\title{
Usefulness of free-breathing readout-segmented echo-planar imaging (RESOLVE) for detection of malignant liver tumors: Comparison with single-shot echo-planar imaging (SS-EPI)
}

\author{
Hirokazu Tokoro $^{a}$, Yasunari Fujinaga ${ }^{a}, *$, Ayumi Ohya ${ }^{a}$, Kazuhiko Ueda ${ }^{a}$, Aya Shiobara ${ }^{b}$, \\ Yoshihiro Kitou $^{\mathrm{b}}$, Hitoshi Ueda ${ }^{\mathrm{b}}$, Masumi Kadoya ${ }^{\mathrm{a}}$ \\ a Department of Radiology, Shinshu University School of Medicine, 3-1-1 Asahi, Matsumoto 390-8621, Japan \\ ${ }^{\mathrm{b}}$ Radiology Division of Shinshu University Hospital, 3-1-1 Asahi, Matsumoto 390-8621, Japan
}

\section{A R T I C L E I N F O}

\section{Article history:}

Received 25 March 2014

Received in revised form 10 June 2014

Accepted 14 June 2014

\section{Keywords:}

Echo-planar imaging

Diffusion magnetic resonance imaging

Abdomen

Liver neoplasms

\begin{abstract}
A B S T R A C T
Purpose: We aimed to clarify the usefulness of free-breathing readout-segmented echo-planar imaging (RESOLVE), which is multi-shot echo-planar imaging based on a 2D-navigator-based reacquisition technique, for detecting malignant liver tumor.

Materials and methods: In 77 patients with malignant liver tumors, free-breathing RESOLVE and respiratory-triggered single-shot echo-planar imaging (SS-EPI) at 3-T MR unit were performed. We set a scan time up to approximately $5 \mathrm{~min}(300 \mathrm{~s})$ before examination, measured actual scan time and assessed (1) susceptibility and (2) motion artifacts in the right and left liver lobes (3, no artifact; 1 , marked), and (3) detectability of malignant liver tumors (3, good; 1 , poor) using a 3-point scale.

Results: The median actual scan time of RESOLVE/SS-EPI was 365/423 s. The median scores of each factor in RESOLVE/SS-EPI were as following in this order: (1) 3/2 (right lobe); 3/3 (left lobe), (2) 2/3 (right lobe); $1 / 2$ (left lobe), and (3) 3/3, respectively. Significant differences were noted between RESOLVE and SS-EPI in all evaluated factors $(P<0.05)$ except for susceptibility of left lobe and detectability of the lesions. Conclusion: Despite the effect of motion artifacts, RESOLVE provides a comparable detectability of the lesion and the advantage of reducing scanning time compared with SS-EPI.
\end{abstract}

(C) 2014 Elsevier Ireland Ltd. All rights reserved.

\section{Introduction}

Single-shot echo planar imaging (SS-EPI) is widely used as diffusion-weighted imaging (DWI) for the identification of focal liver lesions, and is known to be useful for the detection of malignant liver tumors [1-4]. However, SS-EPI has some problems, such as geometric distortion (particularly at tissue-air boundaries) and $\mathrm{T}^{*}$-induced blurring mainly governed by slow traversal through $k$-space [4-11]. Furthermore, these artifacts become even more prominent with an increase in static magnetic field strength.

Multi-shot echo-planar imaging (MS-EPI), in which $k$-space is divided into several segments, can reduce the effect of susceptibility and $\mathrm{T}_{2}^{*}$ decay by accelerating the $k$-space traversal along the phase-encoding direction. On MS-EPI, motion during the diffusion-sensitizing gradients leads to a spatially-dependent phase variation and this causes a high level of artifacts. To

\footnotetext{
* Corresponding author. Tel.: +81 26337 2650; fax: +81 263373087 .

E-mail address: fujinaga@shinshu-u.ac.jp (Y. Fujinaga).
}

compensate for this phase variation and to prevent motion artifacts, navigator echo acquisitions were introduced to monitor shot-toshot phase changes [9-12]. However, conventional MS-EPI, i.e. interleaved EPI, diffusion-weighted data may partially overlap in the phase-encoding direction, leading to gaps in $k$-space that cause ghosting artifacts in the image $[5,8,9]$. For this reason, interleaved EPI is used mainly in organs at rest such as the brain or spinal region $[6,10,13]$.

Readout-segmented echo-planar diffusion-weighted imaging (RESOLVE) is a kind of MS-EPI sequence that divides $k$-space into several segments along the direction of the readout [5,7-9]. On RESOLVE, each segment is densely sampled in the phase-encoding direction as SS-EPI. A short range of readout direction $\left(k_{x}\right)$ in each segment can reduce echo spacing and geometric distortion (susceptibility artifacts). In addition, phase correction between segments is performed using navigator echo and a navigator-based reacquisition technique, which provide a robust correction for motion-induced phase artifacts.

RESOLVE is clinically available and provides data with high resolution and reduced levels of susceptibility artifacts in organs at rest, 
especially in the head [5] and breast region [14]. To our knowledge, the usefulness of RESOLVE under free breathing has been unclear in restless organs such as the liver. We therefore aimed to investigate the advantages and disadvantages of RESOLVE under free breathing for detecting malignant liver tumors.

\section{Materials and methods}

This prospective study was approved by our institutional ethics committee and written informed consent was obtained from all participants.

\subsection{Patients}

Between April 2012 and November 2012, 77 patients (52 men and 25 women; age range, $28-86$ years old; mean age, 68.5 years old), who had malignant liver tumors and underwent magnetic resonance imaging (MRI) including both respiratory-triggered SS-EPI and free-breathing RESOLVE sequences, were enrolled in this study.

Malignant liver tumors comprised hepatocellular carcinoma (HCC) (51 patients), metastasis (19 patients), intrahepatic cholangiocarcinoma (5 patients), primary liver carcinoid (1 patient), and lymphoma (1 patient). The primary sites of liver metastases were the colon ( 7 patients), pancreas (4 patients), lung ( 2 patients), esophagus, ovarian, breast, bile duct, stomach, and vulvar (1 patient). To prevent case selection bias, a maximum of 5 lesions were selected in decreasing order of tumor size when patients had multiple malignant liver lesions. A total of 135 lesions were assessed, made up of 79 HCCs in 51 patients, 41 metastases in 19 patients, 5 intrahepatic cholangiocarcinomas in 5 patients, 5 carcinoid lesions in one patient, and 5 lymphoma lesions in 1 patient. Long axis of the tumor was measured and regarded as the tumor diameter. Mean tumor diameter and standard deviation was $16 \pm 19 \mathrm{~mm}$ (range of diameter: $2-180 \mathrm{~mm}$ ).

Surgical resection was performed in 19 patients with HCC, 2 with a metastatic liver tumor, 4 with intrahepatic cholangiocarcinoma. Twenty-two HCCs, two metastases and 4 intrahepatic cholangiocarcinomas were pathologically proven. In the patient with malignant lymphoma, the pathological diagnosis was obtained with a biopsy. In the remaining patients, tumors were diagnosed based on CT and MR findings (including follow-up studies), as well as clinical information (including existence of a primary malignant tumor and elevation of tumor markers).

\subsection{MRI data acquisition}

All images were obtained with a 3-T MR unit (Trio Tim, Siemens Healthcare, Erlangen) by using a standard body array coil and a spine matrix coil provided by the manufacturer.

Routine MRI examinations were performed, including axial T2weighted imaging [half-Fourier acquisition single-shot turbo spinecho (HASTE) and fat-suppressed fast spin-echo (FSE) sequence] and axial T1-weighted gradient-echo (GRE) imaging, followed by free-breathing RESOLVE and respiratory-triggered SS-EPI. Dynamic contrast-enhanced T1-weighted MRI with fat-suppression (using gadoxetic acid) was performed as necessary.

In this study, free-breathing EPI was not used because respiratory-triggered EPI was recognized as an optimal DWI technique for detecting malignant tumor [15] and could demonstrate focal liver lesions, even when the diameter of lesions was $10 \mathrm{~mm}$ or less [16]. Imaging parameters of RESOLVE were as follows: $\mathrm{TR} / \mathrm{TE}=3500-4500 / 58-60 \mathrm{~ms} ; \mathrm{b}$ factor $=50$ and $800 \mathrm{~s} / \mathrm{mm}^{2}$; field of view $(\mathrm{FOV})=18-31 \times 36-50 \mathrm{~cm}$; number of average $=4$; acquisition matrix $=64-96 \times 128$, and slice thickness/gap $=5 / 1 \mathrm{~mm}$ Imaging parameters of SS-EPI were as follows: $\mathrm{TR} / \mathrm{TE}=2475-6354 / 61-73 \mathrm{~ms} ; \quad \mathrm{b}$ factor $=50$ and $800 \mathrm{~s} / \mathrm{mm}^{2}$;
FOV $=19-31 \times 36-50 \mathrm{~cm}$; number of average $=5$; acquisition matrix $=64-96 \times 128$; slice thickness $/$ gap $=5 / 1 \mathrm{~mm}$ Because we set the scan time for approximately $5 \mathrm{~min}$ in RESOLVE and SS-EPI, some parameters, such as TR, TE, FOV and acquisition matrix, were varied in accordance with patients' body size.

\subsection{Comparison between RESOLVE and SS-EPI}

Although an acquisition time of approximately $5 \mathrm{~min}$ (300 s) was set in each sequence, the actual (required) scan time ranged widely depending on a patient's respiratory cycle. Therefore, we measured the actual scan time of each patient manually to compare the efficiency of RESOLVE and SS-EPI quantitatively.

Image quality was assessed qualitatively based on factors, such as susceptibility artifact, motion artifact and detectability of malignant liver tumors, by two abdominal radiologists (HT and YF, with 5 and 20 years of experience, respectively). All MR images were reviewed with a commercial software package (EV Insite; PSP corporation, Tokyo). Two readers were blind to patient identifiers and clinical information and independently assessed factors in the right and left lobes of the liver.

Susceptibility artifacts were assessed by a 3-point scale based on parenchymal distortion (score 3, no distortion; score 2, arc shaped hyperintensity with slight distortion was seen [moderate]; score 1, obvious distortion was seen [marked]).

Motion artifacts were also assessed by a 3-point scale based on the degree of blurring (score 3, every segmental portal branch was clearly visible [no artifact]; score 2 , segmental portal branches were partially visible [moderate]; score 1 , no segmental portal branches were visible [marked]). If portal veins were occluded or severely compressed, we assessed motion artifacts based on the visibility of right and left hepatic veins.

Detectability of malignant liver tumors was also assessed by a 3point scale (score 3, good; score 2, fair [slight signal change]; score 1, poor). When the score by each reader was not unanimously agreed upon, disagreement was resolved by discussion and consensus.

\subsection{Statistical analysis}

Statistical analyses were performed with a statistical software package (Prism, version 6; GraphPad Software, San Diego, CA). Differences in the actual scan time and the factors of image quality were assessed using a Wilcoxon signed rank test. A $P$ value of $<0.05$ was considered to indicate a statistically significant difference.

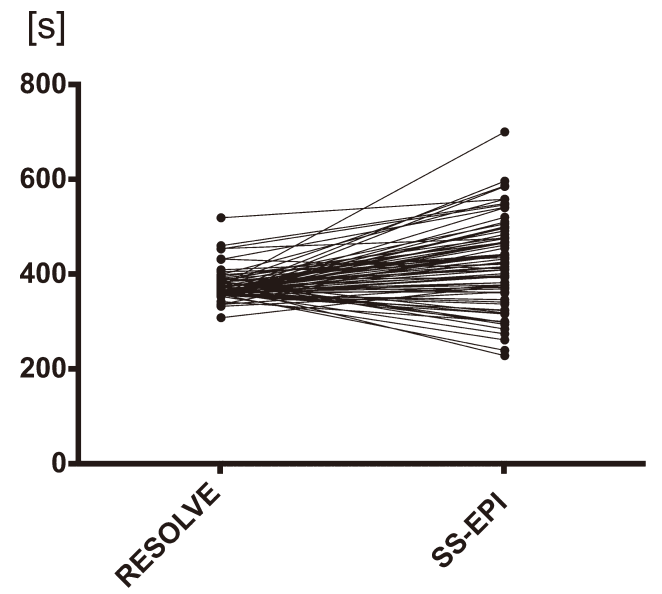

Fig. 1. Actual scan time of readout-segmented echo-planar diffusion-weighted imaging (RESOLVE) and single-shot echo planar diffusion-weighted imaging (SSEPI). 


\section{Results}

Actual scan times of RESOLVE and SS-EPI is shown in Fig. 1. The median and range of actual scan times of RESOLVE and SS-EPI were 365 s (range: 308-519 s) and 423 s (range: 228-700 s), respectively. The median of actual scan time of RESOLVE was significantly shorter than that of SS-EPI $(P<0.0001)$. SS-EPI showed a widely ranging actual scan time, while the time revealed a narrow range in RESOLVE.

Fig. 2 shows representative images for the score of susceptibility artifacts in RESOLVE and SS-EPI. The result of the susceptibility artifact scores in the right lobe (a) and left lobe (b) is shown in Fig. 3. The median of susceptibility artifact scores of RESOLVE and SS-EPI were 3 and 2 (right lobe), 3 and 3(left lobe), respectively. In the right lobe, the mean score of susceptibility artifacts in the RESOLVE group was significantly higher than that in the SS-EPI group $(P<0.0001)$.

Fig. 4 shows representative images for the score of motion artifacts in RESOLVE and SS-EPI. Scores of the motion artifact in the right lobe (a) and left lobe (b) are shown in Fig. 5. The median of motion artifact scores of RESOLVE and SS-EPI were 2 and 3 (right lobe), 1 and 2 (left lobe), respectively. The mean score of motion artifact measurements in the RESOLVE group was significantly lower than that in the SS-EPI group $(P<0.0001)$.

Fig. 6 shows representative images for the score of detectability of malignant liver tumors in RESOLVE and SS-EPI. The result of the lesion detectability scores is shown in Fig. 7. The median of each score of RESOLVE and SS-EPI were 3 and 3, respectively. There was no significant deference on lesion detectability $(P=0.3984)$. Malignant liver tumors were detectable in 92.6\% (right lobe) and 87.8\% (left lobe) in RESOLVE, and 94.7\% (right lobe) and $90.2 \%$ (left lobe) in SS-EPI, respectively. Total detectability was $91.1 \%$ for RESOLVE and 93.3\% for SS-EPI. When the tumors were divided into three subgroups (small, 1-10 mm; medium, 11-20 mm; large, $>20 \mathrm{~mm}$ ) based on the tumor diameter, the detectability of the RESOLVE tended to be less than or equal to that of SS-EPI (Table 1).
However, there was no significant deference of the scores between RESOLVE and SS-EPI in all of the subgroups as well as in the whole group (Table 2). In the large-diameter subgroup, the scores of all the tumors were 3 in both RESOLVE and SS-EPI.

\section{Discussion}

While setting a scan time of $5 \mathrm{~min}$, the median of the actual scan time of RESOLVE was about $6 \mathrm{~min}$, which was approximately $1 \mathrm{~min}$ shorter than that of SS-EPI (Fig. 1). Furthermore, SS-EPI showed a widely ranged actual scan time from about 228-700s, while the range of actual scan time was definitely limited in RESOLVE (Fig. 1). We attributed this result to the difference in the presence or absence of a respiratory-triggering technique. On a respiratorytriggered sequence, image acquisition takes place only within a particular phase of the respiratory cycle. For this reason, variation of the respiratory rates and cycles affect the actual scan time that is ranged widely and prolonged. On the other hand, a RESOLVE sequence is performed without a respiratory-triggered method, i.e. using the free-breathing method in this study, and image acquisition takes place within the whole phases of the respiratory cycle. Therefore, there was little variation of actual scan time among each patient in RESOLVE. This result indicates that RESOLVE has an advantage in improving the efficiency of a routine MR examination. Recently, Choi et al. reported that there was no difference in signal-to-noise ratio (SNR) and contrast-noise ratio (CNR) between navigator-triggered and free-breathing DWI on $3 \mathrm{~T}$ MRI, and freebreathing DWI was more time efficient than navigator-triggered DWI [17]. However, numbers of signal averaging in their sequences were different (one in breath-hold, two in navigator triggered, and four in free-breathing sequences). In addition, initial scan times in their set up are unknown. Therefore, it remains unclear whether free-breathing DWI has an advantage over navigator-triggered DWI or not.

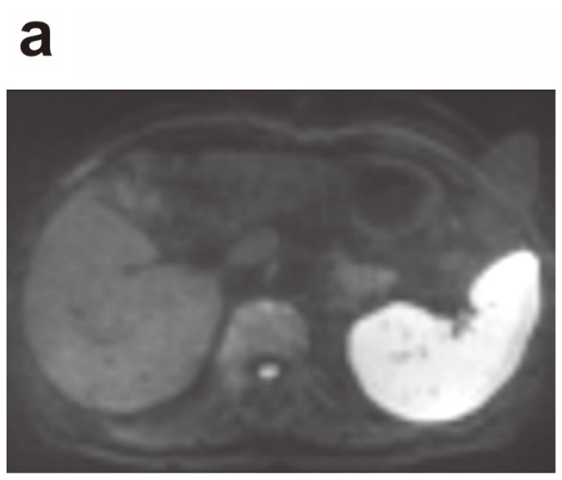

\section{b}

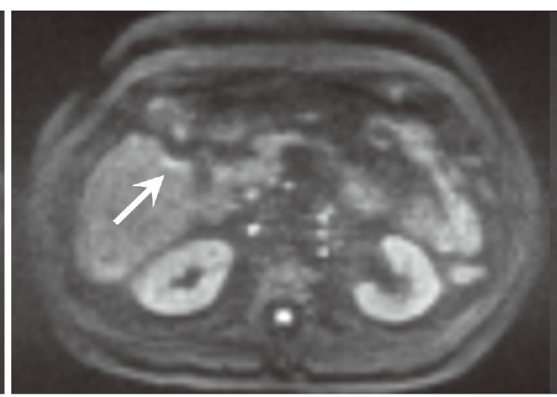

C
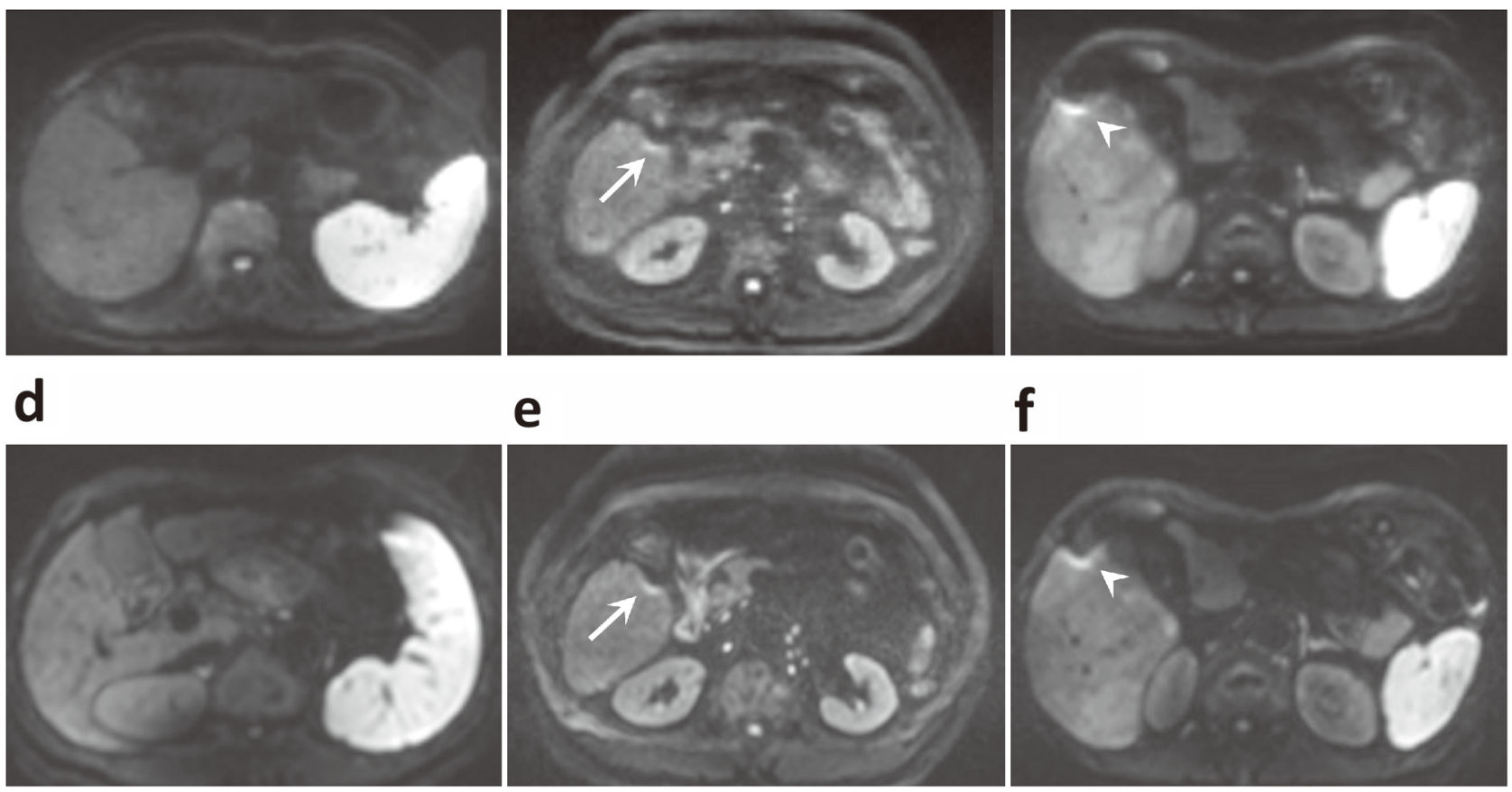
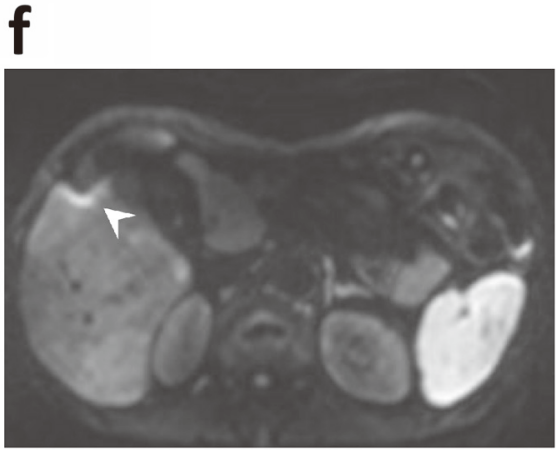

Fig. 2. Representative images for the score of susceptibility artifact. Images of the upper row (a-c) are RESOLVE, and images of the lower row (d-f) are SS-EPI. The leftmost (a, d), center (b, e), and rightmost (c, f) images are scored 3, 2 and 1, respectively. No susceptibility artifact is seen in (a) and (d). Arc shaped high intensity with slight distortion (arrows) is seen at liver-intestine boundaries in (b) and (e). Obvious distortion is seen on liver surface (arrowheads) in (c) and (f). 

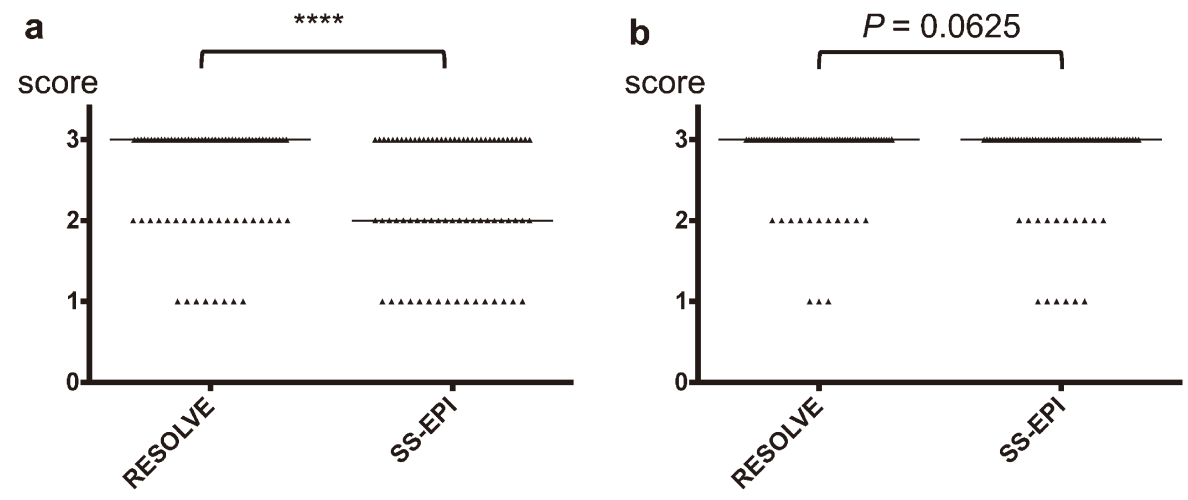

Fig. 3. Score of susceptibility artifact in both lobes of the liver. In the right lobe (a), the score in RESOLVE is significantly higher than that in SS-EPI $(P<0.0001)$, while there is no significant difference in the left lobe $(b)(P=0.0625)$. Long bar, median.
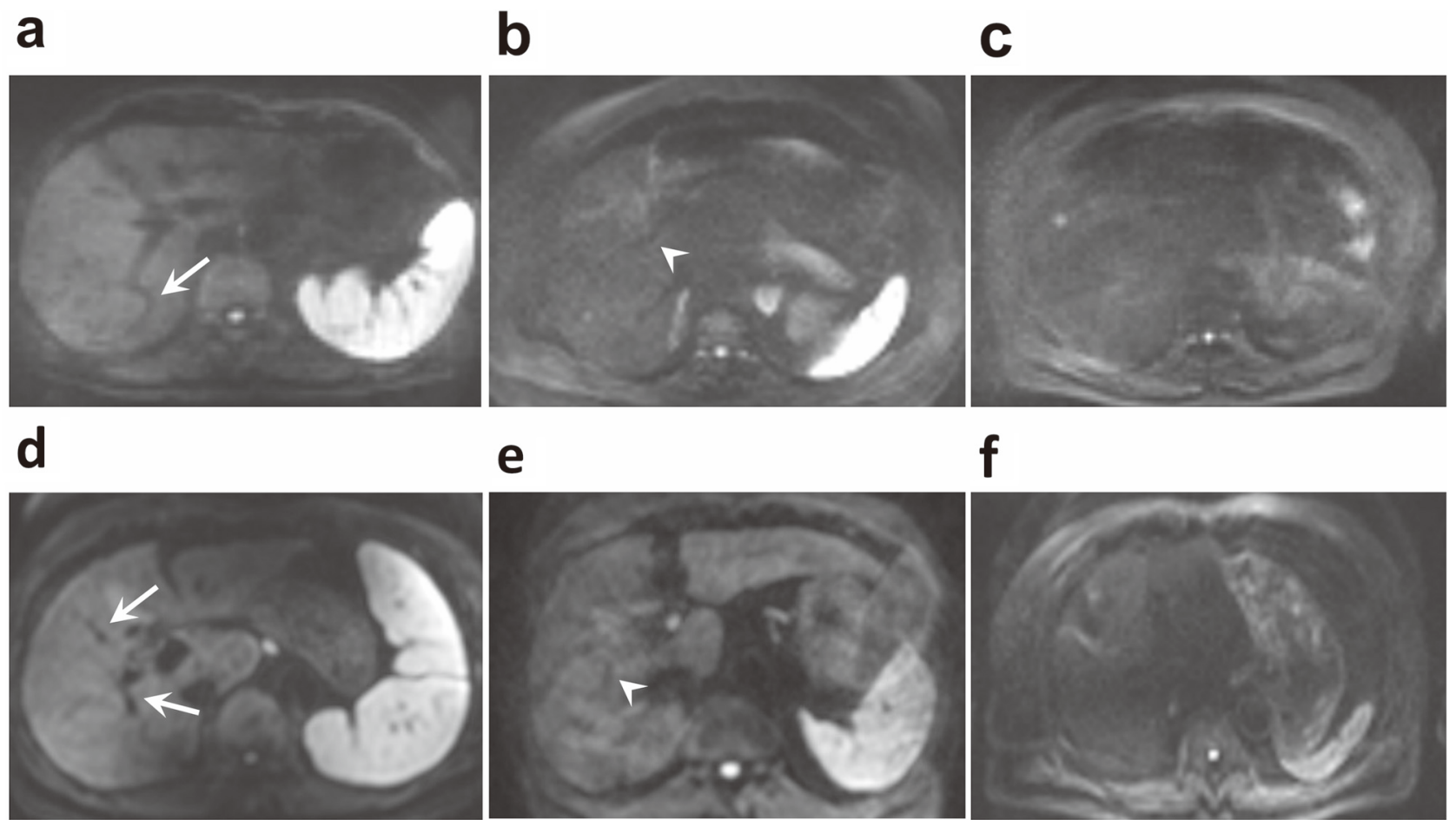

f

Fig. 4. Representative images for the score of motion artifact. Images of the upper row (a-c) are RESOLVE, and images of the lower row (d-f) are SS-EPI. Leftmost (a, d), center (b, e), and rightmost (c, f) images are scored 3, 2, and 1, respectively. All segmental portal branches are clearly seen (arrows) on (a) and (d). Portal branches are detectable but not clear (arrowheads) on (b) and (e). All segmental portal branches of right lobe are undetectable on (c) and (f).
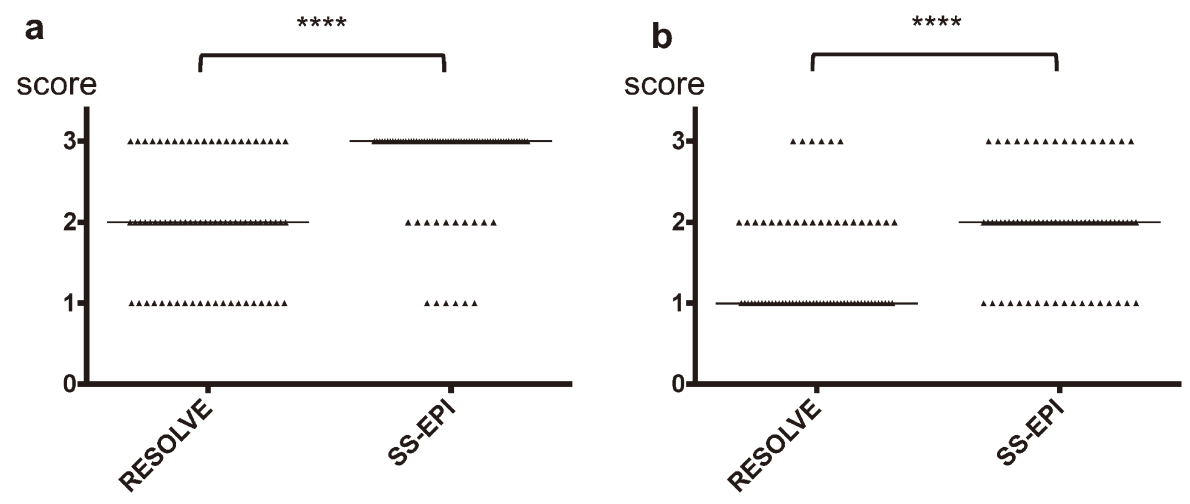

Fig. 5. Score of motion artifact in both lobes of the liver. In the right lobe (a) as well as the left lobe (b), the score in RESOLVE is significantly lower than that in SS-EPI $(P<0.0001)$. 
a

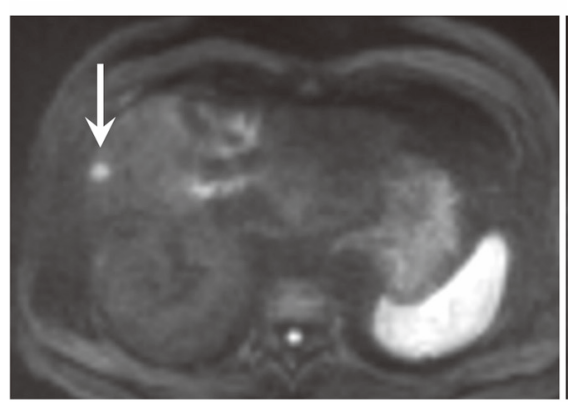

d

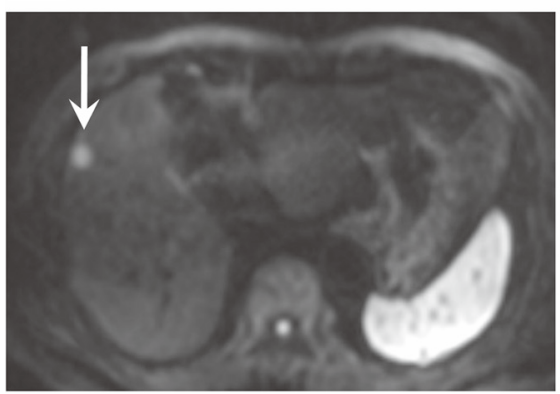

b

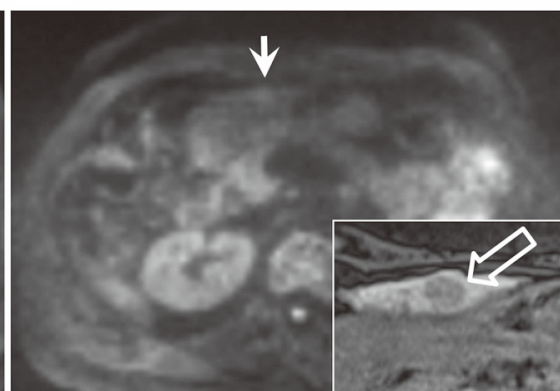

e

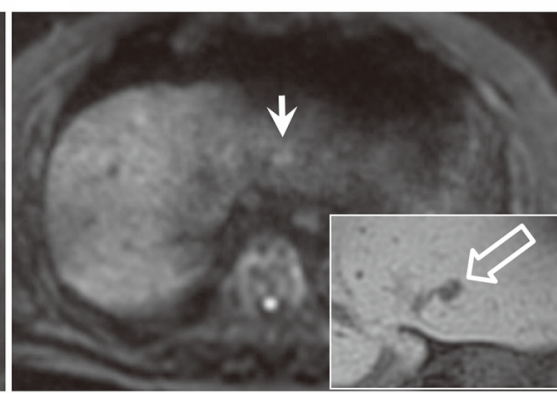

C

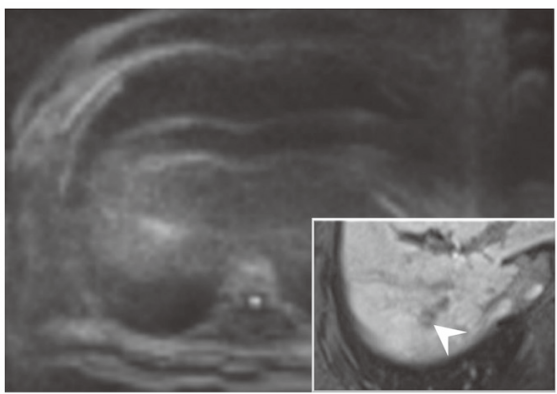

f

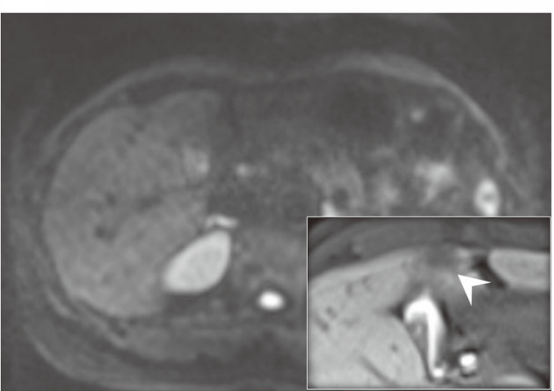

Fig. 6. Representative images for the score of detectability of malignant liver tumors. Images of the upper row (a-c) are RESOLVE, and images of the lower row (d-f) are SS-EPI. The leftmost (a, d), center (b, e), and rightmost (c, f) images are scored 3, 2 and 1, respectively. On RESOLVE (a) and SS-EPI (d) images for score 3, each hepatocellular carcinoma (HCC) is clearly seen as a lesion of marked hyperintensity (long arrows). On RESOLVE (b) and SS-EPI (e) images for score 2, each HCC, that is shown as a lesion of hypointensity on hepatobiliary phase of Gd-EOB-DTPA-enhanced MR image (open arrow in lower right corner image), is shown as a lesion of slight hyperintensity (arrows). On RESOLVE (c) and SS-EPI (f) images for score 1, each tumor (metastatic liver tumor and HCC, respectively) is not detectable, even though the lesion appears hypointense on the hepatobiliary phase of a Gd-EOB-DTPA-enhanced MR image (arrowhead in lower right corner image).

The use of 3-T MR unit enables a higher signal-to-noise ratio. However, it has the disadvantage of being hampered by more pronounced B1 inhomogeneities and dielectric resonances, resulting in more susceptibility artifacts on EPI $[18,19]$. Thus, the reduction of susceptibility is important for obtaining better MR images and detecting malignant lesions on 3 T MR imaging. One of the advantages of MS-EPI is less susceptibility artifacts compared with SS-EPI because of the small amount of accumulating phase error, which was attributed to an effective TE $[6,7,12]$. In this study, a susceptibility artifact in the right lobe was mainly seen around the gallbladder and right colic flexure, and significantly weaker in RESOLVE than
SS-EPI even though the effective TE of RESOLVE was reduced by only about 7.4 ms than that of SS-EPI (Figs. 2 and 3). On the other hand, a susceptibility artifact in the left lobe was not significantly different between RESOLVE and SS-EPI. We attributed this result to other factors, predominantly motion artifacts due to cardiac pulsation.

One of the disadvantages of MS-EPI is a longer acquisition time compared with SS-EPI. MS-EPI is therefore sensitive to motion and likely to be affected by motion artifacts. As mentioned in the introduction, RESOLVE itself has an advantage of lower motion sensitivity than standard MS-EPI. However, motion artifacts were more prominent compared with SS-EPI. We attributed this result

Table 1

Tumor detectability with RESOLVE and SS-EPI.

\begin{tabular}{|c|c|c|c|c|}
\hline \multirow[t]{2}{*}{ Tumor diameter (mm) } & \multicolumn{2}{|c|}{ Number of detected/total lesions with RESOLVE } & \multicolumn{2}{|c|}{ Number of detected/total lesions with SS-EPI } \\
\hline & Right lobe & Left lobe & Right lobe & Left lobe \\
\hline $1-10$ & $34 / 40(85 \%)$ & $9 / 12(75 \%)$ & $36 / 40(90 \%)$ & $10 / 12(83.3 \%)$ \\
\hline $11-20$ & $38 / 39(97.4 \%)$ & $20 / 22(90.9 \%)$ & $38 / 39(97.4 \%)$ & $20 / 22(90.9 \%)$ \\
\hline$>20$ & $15 / 15(100 \%)$ & $7 / 7(100 \%)$ & $15 / 15(100 \%)$ & $7 / 7(100 \%)$ \\
\hline All & $87 / 94(92.6 \%)$ & $36 / 41(87.8 \%)$ & $89 / 94(94.7 \%)$ & $37 / 41(90.2 \%)$ \\
\hline
\end{tabular}

Lesions with the scores 2-3 were considered detected lesions. RESOLVE, readout-segmented echo-planar imaging; SS-EPI, single-shot echo-planar imaging.

Table 2

Comparison of detectability scores between RESOLVE and SS-EPI.

\begin{tabular}{|c|c|c|c|c|c|c|c|c|}
\hline \multirow[t]{2}{*}{ Tumor diameter (mm) } & \multicolumn{2}{|c|}{ Number of lesions } & \multicolumn{2}{|c|}{ Median score with RESOLVE (range) } & \multicolumn{2}{|c|}{ Median score with SS-EPI (range) } & \multicolumn{2}{|l|}{$P$ value } \\
\hline & Right lobe & Left lobe & Right lobe & Left lobe & Right lobe & Left lobe & Right lobe & Left lobe \\
\hline $1-10$ & 40 & 12 & $3(1-3)$ & $3(1-3)$ & $3(1-3)$ & $3(1-3)$ & 0.7500 & $>0.9999$ \\
\hline $11-20$ & 39 & 22 & $3(1-3)$ & $3(1-3)$ & $3(1-3)$ & $3(1-3)$ & $>0.9999$ & 0.5000 \\
\hline$>20$ & 15 & 7 & $3(3)$ & $3(3)$ & $3(3)$ & $3(3)$ & NC & NC \\
\hline All & 94 & 41 & $3(1-3)$ & $3(1-3)$ & $3(1-3)$ & $3(1-3)$ & $>0.9999$ & 0.2500 \\
\hline
\end{tabular}

$P$ values were calculated using a Wilcoxon signed rank test. RESOLVE, readout-segmented echo-planar imaging; SS-EPI, single-shot echo-planar imaging; NC, not calculated. 


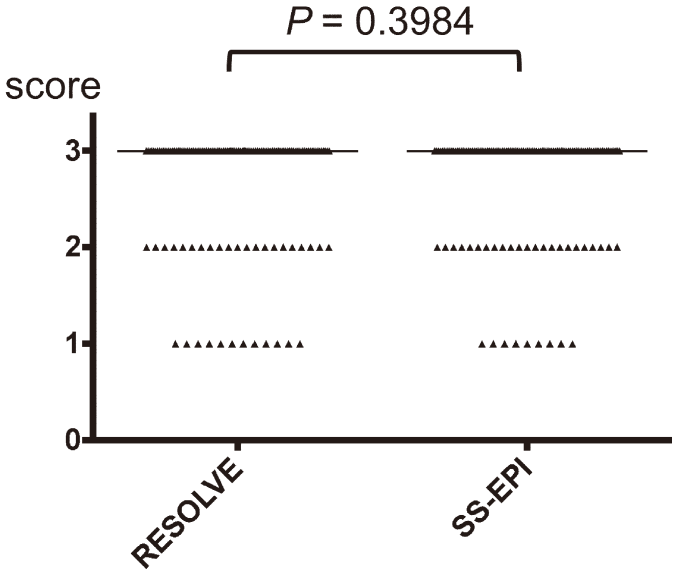

Fig. 7. Score of detectability of malignant liver tumors. There is no significant difference between the scores in RESOLVE and SS-EPI $(P=0.3984)$. Long bar, median.

to the free-breathing method. In this method, the signal intensity of each voxel is influenced by motion-related partial volume averaging. Thus, on free-breathing RESOLVE anatomical details were less clearly defined compared with respiratory-triggered SS-EPI. Our result suggested that RESOLVE sequence including navigator echo and a navigator-based reacquisition technique could not fully reduce motion artifacts due to free breathing.

Despite motion artifacts due to the free-breathing technique, RESOLVE was equal to SS-EPI in detectability regardless of tumor size. Precise tumor staging is necessary for optimal treatment planning, though detection of small focal liver lesions remains a challenging task [16]. Although the visualization of vascular structures in the liver was unsatisfactory in RESOLVE, RESOLVE displayed comparable detectability of tumors to that of SS-EPI even when the tumor size was small (Fig. 7 and Table 2). Takahara et al. reported diffusion-weighted whole-body imaging with background body signal suppression (DWIBS), which is a unique concept of whole-body DWI [20]. This technique intentionally uses free-breathing scanning rather than breath-holding or respiratory-triggered to visualize moving organs and their lesions. The reason for the feasibility of free-breathing in DWI is that diffusion-weighted image contrast is maintained during freebreathing, while breath holding or respiratory triggering reduces image blurring [21]. Therefore, we believe that the free-breathing RESOLVE also has an adequate ability to demonstrate lesions and a potential to provide sufficient image quality as a whole-body DWI.

One of the limitations in this study is that different conditions are adopted for respiratory motion, such as free breathing in RESOLVE and a respiratory-triggered method in SS-EPI. Therefore, the feasibility of RESOLVE under a respiratory-triggered method is unknown for the detection of malignant liver tumors. In addition, a further study comparing RESOLVE and free-breathing SS-EPI is necessary. Another limitation is that some parameters were slightly different between RESOLVE and SS-EPI because the scan time was set for 5 min FOV and acquisition matrix were also varied in accordance with the axial body shape of patients. Furthermore, we did not compare diagnostic accuracy and apparent diffusion conference values between the two sequences, while we focused especially on the detectability of malignant lesions in this study.

In conclusion, despite the effect of motion artifacts, freebreathing RESOLVE provides a comparable detectability of malignant liver tumors and the advantage of reducing scanning time compared with respiratory-triggered SS-EPI.

\section{Conflict of interest}

None of the authors have any actual or potential conflict of interests in relation to this article.

\section{Acknowledgment}

We thank Katsuya Maruyama (Siemens Japan K. K.) for his technical assistance.

\section{References}

[1] Yang DM, Jahng GH, Kim HC, Jin W, Ryu CW, Nam DH, et al. The detection and discrimination of malignant and benign focal hepatic lesions: T2 weighted vs diffusion-weighted MRI. Br J Radiol 2011;84(1000):319-26.

[2] Parikh T, Drew SJ, Lee VS, Wong S, Hechit EM, Babb JS, et al. Focal liver lesion detection and characterization with diffusion-weighted MR imaging: comparison with standard breath-hold T2-weighted imaging. Radiology 2008;246(3):812-22.

[3] Kele PG, van der Jagt EJ. Diffusion weighted imaging in the liver. World J Gastroenterol 2010;16(13):1567-76.

[4] Taouli B, Koh DM. Diffusion-weighted MR imaging of the liver. Radiology 2010;254(1):47-66

[5] Holdsworth SJ, Yeom K, Skare S, Gentles AJ, Barnes PD, Bammer R. Clinical application of readout-segmented-echo-planar imaging for diffusionweighted imaging in pediatric brain. AJNR Am J Neuroradiol 2011;32(7): 1274-9.

[6] Yamashita K, Yoshiura T, Hiwatashi A, Kamano H, Dashjamts T, Shibata S, et al. Detection of middle ear cholesteatoma by diffusion-weighted MR imaging: multishot echo-planar imaging compared with single-shot echo-planar imaging. AJNR Am J Neuroradiol 2011;32(10):1915-8.

[7] Porter DA, Heidemann RM. High resolution diffusion-weighted imaging using readout-segmented echo-planar imaging, parallel imaging and a two-dimensional navigator-based reacquisition. Magn Reson Med 2009;62(2):468-75.

[8] Holdsworth SJ, Skare S, Newbould RD, Bammer R. Robust GRAPPAaccelerated diffusion-weighted readout-segmented (RS)-EPI. Magn Reson Med 2009;62(6):1629-40.

[9] Holdsworth SJ, Skare S, Newbould RD, Guzmann R, Blevins NH, Bammer R. Readout-segmented EPI for rapid high resolution diffusion imaging at $3 \mathrm{~T}$. Eur J Radiol 2008;65(1):36-46.

[10] Atkinson D, Porter DA, Hill DL, Calamante F, Connelly A. Sampling and reconstruction effects due to motion in diffusion-weighted interleaved echo planar imaging. Magn Reson Med 2000;44(1):101-9.

[11] Miller KL, Pauly JM. Nonlinear phase correction for navigated diffusion imaging. Magn Reson Med 2003;50(2):343-53.

[12] Bammer R. Basic principles of diffusion-weighted imaging. Eur J Radiol 2003;45(3):169-84

[13] Bammer R, Augustin M, Prokesch RW, Stollberger R, Fazekas F. Diffusionweighted imaging of the spinal cord: interleaved echo-planar imaging is superior to fast spin-echo. J Magn Reson Imaging 2002;15(4): 364-73.

[14] Bogner W, Pinker-Domenig K, Bickel H, Chmelik M, Weber M, Helbich TH, et al. Readout-segmented echo-planar imaging improves the diagnostic performance of diffusion-weighted MR breast examinations at 3.0 T. Radiology 2012;263(1):64-76

[15] Kartalis N, Loizou L, Edsborg N, Segersvard R, Albiin N. Optimising diffusionweighted MR imaging for demonstrating pancreatic cancer: a comparison of respiratory-triggered, free-breathing and breath-hold techniques. Eur Radiol 2012;22(10):2186-92.

[16] Holzapfel K, Bruegel M, Eiber M, Ganter C, Schuster T, Heinrich P, et al. Characterization of small $(</=10 \mathrm{~mm})$ focal liver lesions: value of respiratory-triggered echo-planar diffusion-weighted MR imaging. Eur J Radiol 2010;76(1):89-95.

[17] Choi JS, Kim MJ, Chung YE, Kim KA, Choi JY, Lim JS, et al. Comparison of breathhold, navigator-triggered, and free-breathing diffusion-weighted MRI for focal hepatic lesions. J Magn Reson Imaging 2013;38(1):109-18.

[18] Galea N, Cantisani V, Taouli B. Liver lesion detection and characterization: role of diffusion-weighted imaging. J Magn Reson Imaging 2013;37(6):1260-76.

[19] Naganawa S, Kawai H, Fukatsu H, Sakurai Y, Aoki I, Miura S, et al. Diffusionweighted imaging of the liver: technical challenges and prospects for the future. Magn Reson Med Sci 2005;4(4):175-86.

[20] Takahara T, Imai Y, Yamashita T, Yasuda S, Nasu S, Van Cauteren M. Diffusion weighted whole body imaging with background body signal suppression (DWIBS): technical improvement using free breathing, STIR and high resolution 3D display. Radiat Med 2004;22(4):275-82.

[21] Kwee TC, Takahara T, Ochiai R, Nievelstein RA, Luijten PR. Diffusion-weighted whole-body imaging with background body signal suppression (DWIBS): features and potential applications in oncology. Eur Radiol 2008;18(9): 1937-52. 